\title{
Normativa laboral para adolescentes en el Perú y la eficacia normativa
}

\section{Labor regulations in Peru for adolescents, and regulatory effectiveness}

\author{
Magali Beatriz Bobadilla Yzaguirre ${ }^{1}$ \\ Universidad Científica del Sur. Lima, Perú \\ mbobadilla@ucientifica.edu.pe / magalibobadilla@gmail.com \\ ORCID 0000-0002-2882-0575
}

Citar como: Bobadilla, M. (2020). Normativa laboral para adolescentes en el Perú y la eficacia normativa. Desde el Sur, 12(1), pp. 127-139.

\section{RESUMEN}

El presente estudio quiere exponer la realidad peruana del adolescente trabajador. Los adolescentes trabajadores con 14 años son objeto de protección normativa desde hace aproximadamente 100 años en el Perú y, a pesar de que nuestras normas de protección han sido armonizadas al contexto internacional a través de los convenios internacionales, no se ha logrado un adecuado ejercicio de los derechos por los interesados, lo que genera informalidad. Todo esto a pesar de contar con normas jurídicas válidas que cumplen los supuestos de eficacia, pero que en la realidad no ofrecen el progreso anhelado.

\section{PALABRAS CLAVE}

Trabajo adolescente, derechos laborales, eficacia normativa, interés superior del menor

\footnotetext{
1 Magíster en Dirección de Personas por la Universidad del Pacífico (Perú) y magíster en Desarrollo Organizacional por la Universidad del Desarrollo (Chile). Doctoranda en Derecho por la Universidad de Salamanca España (DEA, 2003). Abogada por la Universidad Inca Garcilaso de la Vega (Perú), título homologado por la Universidad de Sevilla (España). Abogada colegiada por el ilustre Colegio de Abogados de Madrid y colegiada por Colegio de Abogados de Lima. Miembro del Comité de Calidad de la carrera de Derecho de la Universidad Científica del Sur. Docente de los cursos Derecho Laboral General y Derecho Laboral de la Empresa en la Universidad Científica del Sur y otras universidades. Lidera el proyecto RSU y el la carrera de Derecho de la Universidad Científica del Sur denominado «Soy joven: tengo derecho al trabajo». Se le ha reconocido en la institución por su compromiso en el programa y propiciar agentes de cambio (2019).
} 


\section{ABSTRACT}

The aim of this study is to expose the reality of adolescent labor in Peru. Adolescent workers aged fourteen have been the object of regulatory protection for around one hundred years in Peru; however, although our standards of protection have been brought in line with the global context through international conventions, an adequate exercise of rights on the part of interested parties has not been achieved, and the result has been the generating of an informal labor market. This has occurred in spite of the existence of valid legal norms that meet accepted standards of effectiveness, but which in reality do not offer the progress being sought.

\section{KEYWORDS}

Teenage labor, employment rights, regulatory effectiveness, best interests of the child

«Una buena ley no es aquella que señala unos objetivos justos, sino aquella que además los consigue».

Albert Calsamiglia

\section{Introducción}

El trabajo realizado por niños y adolescentes no es extraño para la sociedad peruana. Desde hace muchos años ha sido considerado, en algunas familias, como un vital apoyo económico. En las zonas rurales del Perú son los hijos quienes tradicionalmente, desde corta edad, colaboran con los cultivos y la crianza de ganado, en un trabajo familiar y no remunerado. Posteriormente, en la adolescencia, estos menores migran a las ciudades en busca de trabajo dependiente para ayudar a sus familias. Entre los más frecuentes están el servicio doméstico en las mujeres y hasta los más riesgosos para los hombres, como es el caso de la minería, la construcción, entre otros (Neves, 2001).

Investigaciones sobre el tema señalan que el Perú (28\%), Ecuador (34\%) y Bolivia (25\%) presentan las tasas más altas del mundo de menores trabajando (Murillo y Román, 2014). Estas cifras, de acuerdo con los resultados de la Encuesta Nacional de Hogares (ENAHO) 2017-2018, nos advierten que 241998 adolescentes de entre 13 y 17 años realizan trabajos que exceden las 50 horas semanales, lo cual se considera como trabajo peligroso (Ontier, 2018). Esta cifra no es compatible con la realidad jurídica laboral de nuestro país, ya que existe una adecuada protección sobre los derechos laborales de los adolescentes en el Perú. 
Desde 1993, el Perú regula, a través de la última versión del Código de los Niños y Adolescentes (CNA), el régimen del adolescente que trabaja. Desde entonces, todo menor de edad a partir de los 12 años puede ejercer su derecho al trabajo dependiente, con respaldo absoluto de la ley, que garantiza los derechos laborales de jornada, como descansos y beneficios sociales. Incluso otorga capacidad para el ejercicio de sus derechos laborales y hasta presentan capacidad procesal laboral, siempre que se encuentre frente a labores ligeras.

La normativa peruana, en cuanto al régimen laboral de los adolescentes, ha sido respetuosa al aplicar puntualmente las normas internacionales que amparan los derechos laborales en niños y adolescentes en nuestro sistema jurídico, con el objeto de garantizar el respeto de sus derechos y hacer viable la realización de condiciones de vida adecuadas para su desarrollo (Valderrama, 2018). Sin embargo, observamos que en 2017 el Estado peruano se interesó por supervisar el cumplimiento de las normas que busquen una adecuada protección aprobando el Protocolo 002-2017-SUNAFIL/INII, denominado «Protocolo de actuación en materia de trabajo infantil» (Sunafil, 2017).

La realidad social indica que muchos de estos jóvenes siguen laborando de manera informal y nociva para su salud e incluso hacen creciente el problema de la deserción del sistema educativo en muchos de ellos. De esta manera, no se cumple el principio del interés superior del menor que garantiza el ordenamiento jurídico, y que en el fondo busca que cada Estado, y la sociedad en su conjunto, debe asegurar que los niños y adolescentes crezcan y se desarrollen con dignidad. Frente a esta situación, nos interrogamos: ¿la normativa laboral para adolescentes en el Perú cumple con el presupuesto de eficacia normativa?

\section{Justificación}

Desde la última versión del Código de los Niños y Adolescentes y demás normas jurídicas, estas regulan el trabajo adolescente en el Perú. Asimismo, de acuerdo con diversas investigaciones el número de adolescentes que trabaja en nuestro país se ha incrementado, y se considera una de las cifras más altas en Latinoamérica.

Con este trabajo, se trata de demostrar que la sola promulgación y fiscalización de normas jurídicas no es suficiente para que sean eficaces en nuestra sociedad. Necesitamos que los sujetos involucrados en esta relación jurídica (adolescentes y empleadores) conozcan las normas para disminuir la tasa de informalidad de estos contratos. Por un lado, los adolescentes, sujetos de esta relación jurídica, son considerados sujetos de protección especial por el Estado. Por otro, tenemos al empresario, que 
es fiscalizado por el Estado con el fin de promover el cumplimiento de las condiciones legales de trabajo de los menores.

Por ello, se realizó un trabajo de campo denominado «Soy joven: tengo derecho al trabajo» para obtener información directa de los jóvenes entre 14 y 17 años, sobre si conocen el marco normativo laboral acerca del trabajo adolescente en el Perú y, por ende, sus derechos laborales. Otro objetivo era informarles sobre sus derechos laborales, porque así construimos un armonioso marco normativo sin que los beneficiarios conozcan de su existencia.

Este trabajo de campo nos permitió analizar que, si bien tenemos leyes armoniosas con el contexto internacional, no logramos sus objetivos con el simple hecho de promulgarlas, sino que debemos buscar alternativas que ayuden a resolver esta situación.

\subsection{Normativa sobre protección laboral del menor}

El marco normativo internacional a través de tratados y convenios ampara los derechos laborales de los adolescentes. Entre ellos tenemos la Convención Americana sobre Derechos Humanos, el Pacto Internacional de Derechos Económicos Sociales y Culturales, y la Convención de los Derechos del Niño. Todos estos documentos normativos destacan sus medidas proteccionistas hacia los menores, y enfatizan que la Convención de los Derechos del Niño es uno de los documentos normativos con mayor aceptación en la comunidad internacional.

Conjuntamente a estos tratados internacionales, la Organización Internacional del Trabajo (OIT) presentó directamente dos convenios que son la base de las normas relativas a los derechos trabajo en el caso de adolescentes. En primer lugar tenemos el Convenio 138 sobre la edad mínima (OIT, 1976) y la Recomendación 146 (OIT, 1973), que determinan las reglas para establecer una edad mínima de trabajo, fijada en 15 años. Sin embargo, esta recomendación señaló que, por excepción en los Estados cuya economía no sea desarrollada, la edad permitida al trabajo es de 14 años. Particularmente, el Perú adoptó la edad de 12 años como edad mínima de admisión al empleo, de acuerdo con la autorización existente en el artículo 32A de la Convención de los Derechos del Niño, siempre que la participación en el trabajo sea ligero. Por ello, los adolescentes peruanos mantienen esta autorización de los 12 años como edad mínima de trabajo y que la Convención 138 (artículos 2, 4 y 7) adicionalmente avala cuando la economía y los medios de educación son insuficientemente desarrollados en el Estado que requiera la autorización. Esta situación era acorde al contexto económico que atravesaba el Perú en 1973 y que, por supuesto, no coincide con el actual. 
Por otro lado, el Convenio 182 (OIT, 1999) busca erradicar internacionalmente las peores formas de trabajo infantil y, de esta manera, considerar la educación como la forma más eficaz de eliminar el trabajo de menores. Este convenio fue ratificado por el Perú el 10 de enero de 2002.

Además, se han incorporado los tratados y las recomendaciones de la OIT a nuestra legislación con mayor interés desde los años 90 . Sin embargo, antes de esa década ya existían normas que posibilitaban el trabajo adolescente en el Perú. Tal es el caso de la Ley 2851 (1918), Ley de Trabajo de Mujeres y Menores, que fijaba la edad mínima de acceso al trabajo en 14 años; la Ley 13968, Código de Menores (1962), y el Código Civil (1936), en cuanto a la posibilidad del permiso de los padres y que actualmente se mantiene.

En el siguiente cuadro apreciamos un resumen de las normas laborales vigentes a la fecha, que rigen los principales derechos de los adolescentes peruanos que trabajan.

TABLA 1. Cuadro comparativo de normas legislativas vigentes sobre protección de los derechos laborales de los adolescentes en el Perú

\begin{tabular}{|c|c|c|c|c|}
\hline $\begin{array}{l}\text { Nor- } \\
\text { ma } \\
\text { legal }\end{array}$ & $\begin{array}{c}\text { Promulga- } \\
\text { ción }\end{array}$ & $\begin{array}{l}\text { Arti- } \\
\text { cullo }\end{array}$ & Objetivo & Comentario \\
\hline $\begin{array}{l}\text { Decreto } \\
\text { Legis- } \\
\text { lativo } \\
295 \\
\text { Código } \\
\text { Civil }\end{array}$ & 1984 & $\begin{array}{l}\text { Art. } \\
457\end{array}$ & $\begin{array}{l}\text { Autori- } \\
\text { zación al } \\
\text { adoles- } \\
\text { cente para } \\
\text { trabajar por } \\
\text { los padres }\end{array}$ & $\begin{array}{l}\text { El Código Civil señala, como requisito indis- } \\
\text { pensable para que los adolescentes puedan } \\
\text { acceder a trabajar, la autorización de los } \\
\text { padres. Esta norma se complementa con las } \\
\text { facultades que otorga el CNA (Varsi, s.f.; Val- } \\
\text { derrama, 2018). }\end{array}$ \\
\hline $\begin{array}{l}\text { Consti- } \\
\text { tución } \\
\text { Política } \\
\text { del } \\
\text { Perú }\end{array}$ & 1993 & $\begin{array}{l}\text { Art. } 4 y \\
23\end{array}$ & $\begin{array}{l}\text { Protección } \\
\text { especial } \\
\text { del Estado } \\
\text { para los } \\
\text { menores }\end{array}$ & $\begin{array}{l}\text { La Constitución Política del Perú de 1993, a } \\
\text { través de dos artículos, confirma su especial } \\
\text { interés en proteger colectivos vulnerables de } \\
\text { la sociedad, aunque para el tipo de protec- } \\
\text { ción especial no se define claramente (Chi- } \\
\text { rinos, 1997). Así, el artículo } 4 \text { de la Consti- } \\
\text { tución señala especial protección para niños, } \\
\text { adolescentes, madre y anciano, y el artículo } \\
23 \text { presta atención prioritaria al trabajo, ra- } \\
\text { tificando protección especial al adolescente } \\
\text { (Elias, 2005; Valderrama, 2018). }\end{array}$ \\
\hline
\end{tabular}




\begin{tabular}{|c|c|c|c|c|}
\hline $\begin{array}{l}\text { Ley } \\
27337, \\
\text { Código } \\
\text { de los } \\
\text { Niños y } \\
\text { Adoles- } \\
\text { centes }\end{array}$ & 2000 & $\begin{array}{l}\text { Art. } 48 \\
\text { al } 68\end{array}$ & $\begin{array}{l}\text { Dere- } \\
\text { chos del } \\
\text { trabajador } \\
\text { adoles- } \\
\text { cente }\end{array}$ & $\begin{array}{l}\text { El Código del Niño y el Adolescente recono- } \\
\text { cen el derecho de trabajar al adolescente, } \\
\text { siempre que no exista explotación eco- } \\
\text { nómica, riesgo para su vida, no afecte su } \\
\text { educación, salud y desarrollo físico, mental, } \\
\text { espiritual y social (Bernuy, 2014). Es destaca- } \\
\text { ble, dentro de este capítulo, los artículos re- } \\
\text { feridos a la edad (art. 51), jornada de trabajo } \\
\text { (art. 56), remuneración (art. 59), derecho } \\
\text { a la seguridad social (art. 64) y al ejercicio } \\
\text { de derechos colectivos (art. 66) (Egúsquiza, } \\
2011 \text {; Valderrama, 2018). }\end{array}$ \\
\hline $\begin{array}{l}\text { Ley } \\
29497, \\
\text { Nueva } \\
\text { Ley } \\
\text { Proce- } \\
\text { sal del } \\
\text { Trabajo }\end{array}$ & 2010 & Art. 8.1 & $\begin{array}{l}\text { Compare- } \\
\text { cencia al } \\
\text { proceso } \\
\text { laboral del } \\
\text { adoles- } \\
\text { cente sin } \\
\text { necesidad } \\
\text { de repre- } \\
\text { sentante } \\
\text { legal }\end{array}$ & $\begin{array}{l}\text { La Nueva Ley Procesal del Trabajo } 29467 \\
\text { mantiene el presupuesto de la Ley } 26636 \\
\text { respecto de la comparecencia al proceso } \\
\text { laboral por parte de los menores de edad. } \\
\text { Sin embargo, a diferencia de su antecesora } \\
\text { (la Ley 29497), manifiesta que esta compa- } \\
\text { recencia no necesita representante legal y } \\
\text { esta ausencia no interfiere en el avance del } \\
\text { proceso. }\end{array}$ \\
\hline
\end{tabular}

Fuente: Elaboración propia, 2019

\subsection{Interés superior del menor en la normativa peruana}

Las normas internacionales sobre protección de menores se inspiran en la Doctrina de la Protección Integral, que reconoce a los niños y adolescentes una serie de derechos económicos y sociales. Esta doctrina, a su vez, introduce principios básicos que son la no discriminación, el derecho a la vida, la supervivencia y el desarrollo, respeto a la opinión del niño en todos los asuntos que le afecten y el interés superior del niño (Sokolich, 2013). El interés superior del niño es un principio jurídico garantista entendiéndolo como una obligación de la autoridad pública destinada a asegurar la efectividad de los derechos subjetivos individuales (Sardegna, 2012). Este principio busca que todas las medidas concernientes a los niños y adolescentes a ser adoptadas por las instituciones públicas o privadas de bienestar social, los tribunales, las autoridades administrativas o los órganos legislativos, deben tener como suprema consideración el «interés superior». Es decir, como algunos manifiestan, prioridad absoluta y privilegio de trato (Chirinos, 2014), lo que ha sido amparado por la Convención de sobre los derechos del niño y por las Naciones Unidas en 1989. Este principio es señalado por algunos autores como concepto jurídico indeterminado e indefinido por la propia Convención (Sokolich, 2013). 
En el Perú, el Código de los Niños y Adolescentes, Ley 27337, acoge este principio en el título preliminar, artículo IX, lo denomina «interés superior del niño y del adolescente» y señala lo siguiente: «En toda medida concerniente al niño y al adolescente que adopte el Estado a través de los Poderes Ejecutivo, Legislativo y Judicial, del Ministerio Público, los gobiernos regionales, gobiernos locales y sus demás instituciones, así como en la acción de la sociedad, se considerará el Principio del Interés Superior del Niño y del Adolescente y el respeto a sus derechos».

El interés superior del menor es el sustento de la normativa laboral peruana para niños y adolescentes, y se fortalece con lo descrito por el artículo 23 de la Constitución Política del Perú de 1993. Este considera a los menores, a la madre y al discapacitado como objeto de protección especial por el Estado en relación con el trabajo y sus diversas modalidades. De esta forma, cumple el privilegio de trato al menor.

\subsection{Eficacia normativa}

Etimológicamente, eficacia viene del vocablo latín efficere, que deriva del verbo facere, que significa «hacer o lograr». Asimismo, el significado guarda similitud en el idioma inglés con efficacy, cuyo significado es «el poder de producir los resultados esperados» (Mokate, 2000).

Desde la perspectiva de Kelsen se analiza a la norma jurídica liberada de elementos de sociología, psicología y ética, y se considera que la eficacia debe relacionarse con el concepto de validez; porque una norma jurídica es considerada como objetivamente válida cuando tiene un mínimo de eficacia (Ugarte, 1995). Para Kelsen, eficacia es igual a obediencia en la aplicación de las normas y, en este sentido, muchos autores a lo largo del tiempo lo sostienen (Correas, 1992).

En el Perú, no es fácil precisar los contenidos de validez, vigencia y eficacia. Sin embargo, aplicamos el concepto de eficacia, según Kelsen, desde una perspectiva pura del derecho, y lo asociamos a los conceptos de validez y vigencia. En este sentido, para que una norma sea eficaz debe ser de cumplimiento exigible; es decir, debe ser aplicada como un mandato dentro del derecho. Adicionalmente, en una Sentencia del Tribunal Constitucional emitida el 24 de junio de 2004, en el expediente 0021-2003-Al/ TC, al referirse a la eficacia, se señala que, además de la vigencia, las normas deben ser publicadas añadiendo el factor publicación (Rubio, 2005).

Frente al positivismo, el concepto de eficacia encuentra en otros juristas una perspectiva sociológica: buscan que estos conceptos no queden simplemente en la teoría jurídica, sino sean además cercanos a sus objetivos en la sociedad. De otro lado, la eficacia es determinar si una norma es 
cumplida o no por las personas a quienes se dirige o los destinatarios de la norma jurídica. Aquí el fondo del concepto recae sobre los destinatarios de la norma jurídica; esta postura es la que consideramos nos enfocaría en resultados más cercanos a la realidad (Leiva, Aristizábal, Martínez y Muñoz, 2011).

\section{Método}

Este estudio es de tipo básico descriptivo y de diseño simple, debido a que se enfocó en analizar la relación existente entre la normativa laboral para adolescentes en el Perú y la eficacia normativa. Para ello, se realizó un análisis de la normativa internacional y nacional relacionada con el derecho al trabajo de los adolescentes y, de otro lado, un análisis sobre el término eficacia normativa, de acuerdo con la legislación en el Perú, que se limitó a considerar solo a los adolescentes de 12 años a 17 años, debido a que estos son los límites que señala la norma peruana.

Adicionalmente, a través del trabajo de campo «Soy joven: tengo derecho al trabajo» se observó socialmente la eficacia de la normativa de trabajo adolescente en el Perú. Por esta razón, se presentaron dos talleres informativos sobre normativa laboral para adolescentes.

Este proyecto, desarrollado en el marco de la responsabilidad social universitaria y del curso de Derecho Laboral de Empresa de la Universidad Científica del Sur, logró que estudiantes de la carrera de Derecho se capaciten y, a su vez, a través de talleres, instruyan a 104 adolescentes del Instituto Educativo 7213 Peruano Japonés de Villa El Salvador. A través de unas encuestas previas dicotómicas realizadas en esta capacitación, se obtuvo los siguientes resultados: el 76\% de los encuestados manifestó que no conocían las normas laborales que los protegían; el 9\% trabajan dependientemente; $16 \%$ trabajan por su cuenta y, luego de la capacitación y evaluación final, manifestaron reconocer las normas esenciales que los protegen en el ámbito laboral dependiente y sus derechos laborales básicos a través de una breve evaluación.

\section{Resultados}

La normativa sobre derechos laborales adolescentes en el Perú data de 1918. Han transcurrido más de 100 años en los que reconocemos a nuestros adolescentes el derecho al trabajo. Desde hace 20 años, tenemos un sistema jurídico articulado a las normas internacionales que marcaron los estándares mínimos de protección, como la Convención de los Derechos del Niño, los Convenios de la OIT, entre otros de relevancia, pero que mantienen una situación social para los adolescentes peruanos al trabajar en riesgo e informalidad. La brecha es evidente entre la existencia de las 
normas generales armoniosas, válidas, vigentes y publicadas si en la realidad a quien se dirigen desconoce su existencia.

El trabajo dependiente en los menores peruanos es una realidad y va en aumento. Desde la perspectiva formal lo observamos en las estadísticas del Ministerio de Trabajo: en el primer trimestre de 2019 formalmente los niveles de autorización para el trabajo de menores son de 138 autorizaciones a nivel nacional. Por tanto, es de especial interés centrar la atención en este sujeto de trabajo que tiene consideración de ser especialmente protegido por el Estado, de acuerdo con el artículo 23 de la Constitución de 1993 y por el principio de interés superior. Por lo tanto, debemos conocer si las normas de protección realmente cumplen estos objetivos.

El Perú opta por un concepto de eficacia normativa desde una perspectiva pura del derecho, y lo asocia a los conceptos de validez y vigencia. Desde esta perspectiva, las normas referidas a la protección jurídica laboral de los menores son normas eficaces. Todas cumplen el procedimiento de validez y se encuentran vigentes, pero el impacto de la norma en esta realidad es distinto en cuanto a su objetivo, porque observamos adolescentes trabajando ajenos a los derechos que les corresponden y empleadores que desconocen las exigencias mínimas de las condiciones adecuadas para contratar con menores y evitar sanciones.

Por ello, desde otra perspectiva más integradora, el concepto de eficacia se asocia a la sociología jurídica, que propone un análisis más social y destaca que «no basta con que la norma exista formalmente y pueda ser exigida, sino que se cumpla las funciones para las cuales fue creado el Derecho (para que encauce, limite, garantice y eduque) (Leiva, Aristizábal, Martínez y Muñoz, 2011). Este concepto busca justificar las alternativas del derecho en la vida social, debido a la influencia y variación de la sociedad, lo que nos permitiría fijar objetivos claros en base a la investigación del tema.

Para lograr eficacia existen factores que contribuyen al éxito. Dentro de ellos encontramos los presupuestos de eficacia normativa como el conocimiento del derecho, que es una de las más importantes, porque permite conectar entre el concepto normativo y sociológico. A través de este presupuesto, «Nadie puede obedecer lo que no conoce. Por lo tanto, en mérito de la eficacia de la norma jurídica legal, el presupuesto de conocimiento del derecho establecido es inexcusable» (Donzis, 2006). A partir de aquí, es necesario que el Estado y la acción de la sociedad en su conjunto asuman las condiciones materiales para que los ciudadanos cumplan con las normas. Esta postura sería la más efectiva social y jurídicamente para el cumplimiento de las normas laborales de los adolescentes en el Perú. 


\section{Limitaciones}

Este estudio tuvo diversas limitaciones que se deben tener en cuenta para otros estudios. Las estadísticas oficiales que contamos sobre autorizaciones de trabajo en el caso de menores adolescentes actualmente son escasas. De acuerdo con la información de la Oficina de Estadística del Ministerio de Trabajo, durante el primer trimestre de 2019 a nivel nacional se realizaron 138 autorizaciones de trabajo para menores.

Sobre el tema desconocemos la cantidad de menores que laboran realmente, debido a la alta informalidad en el sector trabajo, cuyas cifras rondan el 73\% desde 2016 según el INEI. A partir de ahí nos lleva a cuestionarnos sobre lo siguiente: ¿qué porcentaje les corresponden a nuestros menores? Además, hay que distinguir el trabajo dependiente del independiente en estos casos, ya que la norma laboral solo protege a los adolescentes trabajadores dependientes.

El trabajo de campo se realizó de manera transversal, en un momento único. Ello impidió contar con una población más amplia de estudiantes, lo que redujo el tamaño de la muestra.

\section{Conclusiones}

En el Perú, la normativa laboral dependiente de los adolescentes define el primer segmento social dentro de nuestra legislación laboral. De 12 a 17 años las normas de protección laboral al adolescente establecen derechos acordes a la edad y que deben ser también conocidos y exigibles por los empleadores.

Mantener las condiciones que la legislación peruana respecto a la edad mínima (12 años) en el caso del «trabajo ligero» para acceder al empleo debería ser revisado urgentemente. Con esto se podrá exigir el cumplimiento uniforme de los 14 años como edad de trabajo como mínimo exigible.

Los adolescentes son sujetos especiales de protección; por tanto, debe ser también un derecho esencial para ellos conocer las normas y los derechos que estas contienen y que los protegen, para que no sean vulnerables al momento de integrarse al ámbito laboral. Por esta razón, es importante construir nuestros objetivos e indicadores relacionados con la protección de los derechos laborales de los adolescentes con vistas de mejorar nuestras normas y proyectarlas según nuestra realidad económica y social.

Es indispensable ejecutar proyectos de difusión de la normativa laboral adolescente de alcance nacional, sobre todo en zonas rurales. Por nuestra experiencia a través del proyecto «Soy joven: tengo derecho al trabajo», sabemos que el conocimiento tiene aceptación en los adolescentes. 
En el Perú la eficacia normativa es un tema discutido. Forma parte de los conceptos iniciales de los estudios de Derecho; sin embargo, su definición siempre se confunde con validez y eficiencia. Su utilización positivista no aterriza, en la práctica, un concepto que desde su raíz etimológica busca alcanzar objetivos. Por ello, es importante aplicar una metodología sociojurídica para lograr resultados acordes con nuestra realidad. La eficacia en el Perú se sitúa desde la perspectiva positivista y bajo esa concepción tendríamos que señalar que nuestras normas son eficaces y que han logrado sus objetivos; aunque en la realidad no se ha logrado.

Finalmente, podemos decir que para el peruano ver adolescentes trabajando no es novedad. Para algunos sectores es incluso una conducta positiva frente a la vulnerabilidad de la pobreza, pero olvidamos como sociedad que el adolescente debe centrarse en su educación, y que aquellos adolescentes que laboran cuentan con menos tiempo para sus deberes estudiantiles y enfrentan su jornada escolar más cansados. Esto supone mayor esfuerzo para cumplir las demandas escolares, puede aumentar el riesgo de deserción escolar y, por tanto, truncar su futuro (Murillo y Román, 2014).

\section{Contribución del autor}

La autora ha participado en la concepción integral y la versión final del artículo.

\section{Fuentes de financiamiento}

Propias.

\section{Conflictos de intereses}

Ninguno. 


\section{REFERENCIAS BIBLIOGRÁFICAS}

Chirinos, E. (1997). De los derechos sociales y económicos. En Constitución 1993. Lectura y comentario (pp. 56-57). Lima: Nerman.

Correas, O. (1992). Kelsen o Gramsci o de la eficacia como signo de hegemonía. Recuperado de https://revistas-colaboracion.juridicas.unam.mx/ index.php/critica-juridica/article/download/3025/2826

Donzis, R. (2006). La eficacia social de las normas jurídicas. Revista Electrónica de Teoría y Práctica de la Elaboración de Normas Jurídicas, pp. 6-24.

Egúsquiza, B. (2011). Trabajo de menores de edad. Actualidad Empresarial, 226, pp. 4-6.

Elías, F. (2005). Atención prioritaria del derecho al trabajo. En La Constitución comentada. Lima: Gaceta Jurídica.

Leiva, E., Aristizábal, J., Martínez, J. y Muñoz, A. (noviembre de 2011). Eficacia jurídica y sociológica de los derechos fundamentales de las trabajadoras sexuales en Colombia. Recuperado de https:/dialnet.unirioja.es/ servlet/articulo?codigo $=3896299$

Mokate, K. (2000). Eficacia, eficiencia, equidad y sostenibilidad. Recuperado de https://www.cepal.org/ilpes/noticias/paginas/9/37779/gover_2006_03_eficacia_eficiencia.pdf

Murillo, F. y Román, M. (2014). Consecuencias del trabajo infantil en el desempeño escolar. Latin American Research Review, 49(2), pp. 84-106.

Neves, J (2001). El trabajo infantil en las acciones de interés público. lus et Veritas, 12(23), pp. 224-236.

OIT (17 de junio de 1999). Convenio sobre la prohibición de las peores formas de trabajo infantil y la acción inmediata para su eliminación. C182, Convenio sobre las peores formas de trabajo infantil, 1999. Ginebra: OIT.

OIT (26 de junio de 1973). Recomendación sobre la edad mínima de admisión al empleo. R146, Recomendación sobre la edad mínima, 1973. Ginebra: OIT.

OIT (19 de junio de 1976). Convenio sobre la edad mínima de admisión al empleo. C138, Convenio sobre la edad mínima, 1973. Ginebra: OIT.

Ontier (junio de 2018). Boletín Informativo sobre Novedades en Derecho Laboral. Recuperado de https://pe.ontier.net/ia/boletin-laboral-junio-2018. pdf

República del Perú (23 de noviembre de 1918). Ley 2851. Trabajo de los niños y mujeres por cuenta ajena.

Rubio, M. (2005). La vigencia y validez de las normas jurídicas en la jurisprudencia del Tribunal Constitucional. Themis, 51, pp. 7-18. 
Sardegna, P. (2012). El interés superior del niño trabajador y la protección efectiva de sus derechos en Argentina. Cognitio Juris, 4, pp. 68-79. Recuperado de https://dialnet.unirioja.es/servlet/articulo?codigo=3918154

Sentencia del Tribunal Constitucional (24 de junio de 2004). Expediente 0021-2003-AI/TC sobre acción de inconstitucionalidad interpuesta por el Colegio de Biólogos y el Colegio de Arquitectos del Perú contra la Ordenanza Municipal 006-2002-MPP.

Sokolich, M. (2013). La aplicación del principio del interés superior del niño por el sistema judicial peruano. Vox Juris, 25(1), pp. 81-90.

Sunafil (9 de junio de 2017). Resolución de Superintendencia 114-2007-SUNAFIL. Protocolo 002-2017-SUNAFIL/INII. Lima.

Ugarte, J. (1995). El sistema jurídico de Kelsen. Síntesis y crítica. Revista Chilena de Derecho, 22(1), pp. 109-118.

Valderrama, L. (2018). Trabajo infantil y adolescente. Régimen normativo y fiscalización. Soluciones Laborales, 127, pp. 13-38.

Varsi, E. (s.f.). Autorización para el trabajo del menor. En Código Civil comentado por los mejores especialistas. Tomo III. Lima: Gaceta Jurídica. Recuperado de andrescusi.blogspot.com: https://andrescusi.files.wordpress. com/2014/03/codigo-civil-comentado-tomo-iii.pdf

Recibido: 5/9/2019

Aceptado: 6/11/2019 